\title{
DYNAMIC CONTROLLERS WHICH USE DIFFERENCE APPROXIMATES OF OUTPUT DERIVATIVES AND THEIR PRACTICAL STABILITY
}

\author{
H. Kokame ${ }^{1} \quad$ K. Hirata $^{1} \quad$ T. Mori $^{2}$ \\ 1) Dept. of Electrical \& Electronic Systems, Osaka Prefecture University \\ 2) Dept. of Electronics \& Information Science, Kyoto Institute of Technology
}

\begin{abstract}
The derivative feedback is a classical but representative means in the design of control systems, and for practical reasons it is often replaced by its difference approximation. As the resulting closed-loop system involves a time-delay, it does not necessarily preserve stability however accurate the approximation is. Following the terminology of Palmor (1980), it may be said that the practical stability is not always guaranteed. The present paper studies the condition under which the practical stability is guaranteed for approximating output difference feedback controllers. Copyright (c)2005 IFAC
\end{abstract}

Keywords: output difference feedback, delay differential equation, derivative feedback, practical stability, stabilization

\section{INTRODUCTION}

The derivative feedback is a classical but representative means in the design of control systems, and the derivative is often replaced by its difference approximates in practical situations such that the derivative of output is not measured directly. For this case, the resulting closed-loop system involves a time-delay originated from taking the difference, and sometimes loses the stability however accurate the approximation is. (e.g., Kokame and Mori 2002).

Similar singular phenomena are known to be possible when applying the Smith predictive controller to time-delay systems. In fact Palmor (1980) showed that any small mismatch between the real time-delay of the plant and its estimate employed in the controller might lead to the closed-loop instability. More recently, singularities caused by small errors in realizing numerically the finite spectrum assignment controllers has been reported (Van Assche et al. 1999; Santos and Mondie 2000; Engelborghs et al. 2001). The above singularities are attributed to the fact that errorneous controllers lead to a neutral system. It is added that the robust stabilization of neutral systems is sometimes quite difficult (e.g., O'Connor and Tarn 1983; Yanushevsky 1992).
It is noted however that the difference approximation of derivatives leads to a retarded system. Possible instability when using the difference approximation seems to have evaded the close attention of researchers, while a general result has been provided recently in Kokame and Mori(2002). The result is valid for the case of full state measurement, and the condition is expressed simply in terms of the eigenvalues of the product of the input matrix and the gain matrix. The aim of the present paper is to extend this new result to the case where only partial states are measurable, and discuss the robustness of the difference feedback compared with other approximate controllers.

The paper is organized as follows. Section 2 presents a motivation to use the derivative or difference feedback in nonlinear environments. Further, the difficulty in obtaining a stabilizing difference feedback controller which approximates a derivative feedback controller is described briefly. In Section 3, we describe the problem in the case of output measurements, and introduce some new tools. Section 4 provides the main results, assuming either the full-order observer-based difference feedback or the minimal-order observer-based counterpart. A simple example is added which illustrates the robustness of the difference feedback con- 
troller. Section 5 concludes the paper with some remarks. In the following, the determinant of a matrix $X \in C^{n \times n}$ is denoted by $\operatorname{det}[X]$, and its eigenvalues are denoted by $\lambda_{i}(X), i=1, \ldots, n$.

\section{DERIVATIVE AND DIFFERENCE FEEDBACK}

This section briefly describes the advantage of using either derivative feedback or output difference feedback. Consider a nonlinear system

$$
\dot{x}=f(x, u, p),
$$

where $x \in R^{n}, u \in R^{m}$, and $p \in R^{d}$ are the state, input, and parameter vectors, respectively. Let $x_{p}$ be an unstable fixed point associated with $p$, i.e., $f\left(x_{p}, 0, p\right)=0$. The state deviations $\delta x(t)=x(t)-x_{p}$ are governed by

$$
\delta \dot{x}(t)=A_{p} \delta x(t)+B_{p} u(t),
$$

where $A_{p}$ and $B_{p}$ are Jacobian matrices $\frac{\partial f\left(x_{p}, 0, p\right)}{\partial x}$ and $\frac{\partial f\left(x_{p}, 0, p\right)}{\partial u}$, respectively.

Suppose that the state vector $x(t)$ is measurable, but the parameter $p$ is uncertain. For this case, $\delta x$ is not available, so that the feedback controller $u=-K \delta x$ can not be realized. However, the derivative of $\delta x$ is in principle available from the state measurements, since $\delta \dot{x}(t)=\dot{x}(t)$ holds independently of the value of parameter $p$. Thus the following feedback is of special interest:

$$
u(t)=-K \delta \dot{x}(t)
$$

In case the derivative is not obtainable or desirable, it may be replaced by the difference of $\delta x$,

$$
u(t)=-\frac{1}{T} K(\delta x(t)-\delta x(t-T)) .
$$

Note also that the state difference is obtained from the relation $\delta x(t)-\delta x(t-T)=x(t)-x(t-T)$. Let us denote the linearized system (2) in the case of the nominal $p_{0}$ by

$$
\delta \dot{x}(t)=A \delta x(t)+B u(t),
$$

and suppose that it is stabilized by either the derivative feedback (3) or the difference feedback (4). Then, from continuity, the closed-loop stability will remain for a small change of the parameter vector. It means that as far as the closed-loop stability is maintained, the state $x$ converges to the true steady state $x_{p}$. This desirable property may be compared to the usefulness of the integrator in servo systems. It is also noted here that in the physics community the difference feedback is well known under the name of the delayed feedback, since the invention by Pyragas (1992).

A problem arises with the difference feedback, as it makes a delay-differential system of retarded type:

$$
\delta \dot{x}(t)=A \delta x(t)-\frac{1}{T} B K(\delta x(t)-\delta x(t-T)) .
$$

That is, even if the derivative feedback (3) is stabilizing, its differece approximation (4) does not always guarantee the stability of the closed-loop system (6) having small $T$. As for a general fact, Ushio (1996) first pointed it out for discrete-time systems that stabilization via the difference feedback is not always possible. The same limitation was proven for continuoustime systems in Nakajima (1997) and Just et al. (1997). The inherent limitation is immediate from the following lemma (see e.g., Kokame et al., 2001).

Lemma 1 Consider the $n$-th order delay differential system

$$
\delta \dot{x}(t)=A \delta x(t)+H(\delta x(t)-\delta x(t-T)) .
$$

If $\operatorname{det}[-A] \leq 0$, then the system is unstable for any $T>0$. Further there exists a monotone unstable mode.

The determinant assumption of Lemma 1 means that the system has either an eigenvalue on the origin or an odd number of real positive eigenvalues. Hence it is called the odd number condition. Especially in the case where $\operatorname{det}[-A]=0$, it is easy to see that the retarded system (7) always has an eigenvalue on the origin, hence it is not asymptotically stable.

\section{DYNAMIC CONTROLLERS}

Suppose that the nonlinear system (1) is associated with the output vector,

$$
y=h(x, u, p), \quad y \in R^{q} .
$$

The output deviation $\delta y=y-y_{p}, y_{p}=h\left(x_{p}, 0, p\right)$, is

$$
\delta y(t)=C_{p} \delta x(t)+D_{p} u(t)
$$

where $C_{p}=\frac{\partial h\left(x_{p}, 0, p\right)}{\partial x}$ and $D_{p}=\frac{\partial h\left(x_{p}, 0, p\right)}{\partial u}$. Though $\delta y$ is not available as before, we can utilize the derivative of $\delta y$. Consider the dynamic derivative feedback controller,

$$
\begin{aligned}
& \dot{\xi}(t)=A_{c} \xi(t)+B_{c} \delta \dot{y}(t), \\
& u(t)=C_{c} \xi(t)+D_{c} \delta \dot{y}(t),
\end{aligned}
$$

where $\xi$ is assumed to have an adequate dimension. Suppose that the controller (10) stabilizes the nominal model

$$
\begin{aligned}
& \delta \dot{x}(t)=A \delta x(t)+B u(t) \\
& \delta y(t)=C \delta x(t)+D u(t)
\end{aligned}
$$

where $C$ and $D$ respectively denote $C_{p}$ and $D_{p}$ for the nominal $p_{0}$. In the following, we are devoted to the cases where either $D=0$ or $D \neq 0, D_{c}=0$, Furthermore $(A, B)$ and $(A, C)$ are assumed to be controllable and observable, respectively. It is confirmed again that if $p$ changes to $\hat{p}$ in a small neighborhood of $p_{0}$, the state will converge to the corresponding steady state $x_{\hat{p}}$ without offsets. 
In the first place, consider the case $D=0$. For this case, we may take $\delta \dot{y}$ as the output:

$$
\begin{aligned}
& \delta \dot{x}(t)=A \delta x(t)+B u(t), \\
& \delta \dot{y}(t)=C A \delta x(t)+C B u(t) .
\end{aligned}
$$

The above system is observable if and only if $A$ is nonsingular. Thus if $\operatorname{det}[-A] \neq 0$, especially even if $\operatorname{det}[-A]<0$, we can find a stabilizing dynamic controller of the form (10). The closed-loop system is given by

$$
\begin{aligned}
\dot{z}(t) & =\mathcal{A} z(t)+\mathcal{B} v(t) \\
& =\left[\begin{array}{cc}
A & B C_{c} \\
0 & A_{c}
\end{array}\right] z(t)+\left[\begin{array}{c}
B D_{c} \\
B_{c}
\end{array}\right] v(t), \\
v(t) & =-\mathcal{K} \dot{z}(t)=\left[\begin{array}{ll}
C & 0
\end{array}\right] \dot{z}(t),
\end{aligned}
$$

where $z=\left[\begin{array}{ll}\delta x^{T} & \xi^{T}\end{array}\right]^{T}$ and $v$ denotes $\delta \dot{y}$.

Proceed to the second case where $D \neq 0$ and $D_{c}=0$. The closed-loop system is written by

$$
\begin{aligned}
\dot{z}(t) & =\mathcal{A} z(t)+\mathcal{B} v(t) \\
& =\left[\begin{array}{cc}
A & B C_{c} \\
0 & A_{c}
\end{array}\right] z(t)+\left[\begin{array}{c}
0 \\
B_{c}
\end{array}\right] v(t), \\
v(t) & =-\mathcal{K} \dot{z}(t)=\left[\begin{array}{ll}
C & D C_{c}
\end{array}\right] \dot{z}(t),
\end{aligned}
$$

Assuming the closed-loop systems (13) and (14) are stable, we may utilize a difference approximation in place of $v$,

$$
\begin{aligned}
v_{d}(t) & =-\frac{1}{T} \mathcal{K}(z(t)-z(t-T)) \\
& =\frac{1}{T}(\delta y(t)-\delta y(t-T)) .
\end{aligned}
$$

Now we would like to see if such a difference approximation can stabilize for some small $T$. It is noticed here that for both cases, the singularity $\operatorname{det}[-A]=0$ implies $\operatorname{det}[-\mathcal{A}]=0$ again. Thus the zero eigenvalue problem can not be avoided by the use of the dynamic controller (10).

By noting for both cases, the closed-loop systems are expressed as a derivative feedback of the augmeted state, we can apply a recent result of Kokame and Mori (2002). Given the linear time-invariant system,

$$
\delta \dot{x}(t)=A \delta x(t)+B u(t),
$$

suppose that it is stabilized by the derivative feedback

$$
u(t)=-K \delta \dot{x}(t)+v(t) .
$$

The closed-loop system is

$$
\delta \dot{x}(t)=\bar{A} \delta x(t)+\bar{B} v(t),
$$

where $\bar{A}=(I+B K)^{-1} A$ is Hurwitz from the assumption, and $\bar{B}=(I+B K)^{-1} B$. If we employ an approximate difference feedback,

$$
u(t)=-\frac{1}{T} K(\delta x(t)-\delta x(t-T))+v(t),
$$

then the closed-loop system is given by

$\delta \dot{x}(t)=A \delta x(t)-\frac{1}{T} B K(\delta x(t)-\delta x(t-T))+B v(t)$.

The stability of the time-delay system (19) does not follow automatically even if $T$ is chosen to be small enough. Following the terminology of Palmor (1980), the closed-loop system (19) will be said to be practically stable if it is stable for all sufficiently small $T$. From this definition, it is said that the closed-loop system is not practically stable, if there exists an arbitrary small $T$ for which the closed-loop system is unstable. The following lemma is immediate from Theorem 1 and Theorem 3 of Kokame and Mori (2002), and it provides a key to determine if the time-delay system is stable for small $T$.

Lemma 2 Let $\Gamma(s)$ be the complementary sensitivity of the derivative feedback system (17), i.e.,

$$
\Gamma(s)=s K(s I-\bar{A})^{-1} \bar{B},
$$

and let $g(s)$ be an entire function defined by

$$
g(s)=\operatorname{det}\left[I-\left(1-\frac{1-e^{-s}}{s}\right) \Gamma(\infty)\right]
$$

Then, as to the practical stability of the retarded system (19), the followings are valid.

(i) If all the roots of $g(s)$ are located in the open lefthalf-plane, the closed-loop system is practically stable. (ii) If $g(s)$ has a root in the open right-half-plane, the closed-loop system is not practically stable.

From Lemma 2, we know that the practical stability of the difference feedback depends on the high frequence gain $\Gamma(\infty)$. By noting $\Gamma(\infty)=(I+K B)^{-1} K B$, Lemma 2 is rewritten in terms of the eigenvalues of matrix product $K B$, as follows:

Lemma 3 Consider a curve on the complex plane,

$$
\Phi=\left\{\frac{-j \omega}{1-e^{-j \omega}}:-2 \pi<\omega<2 \pi\right\} .
$$

The curve $\Phi$ divides the whole plane into two unbounded regions, $\Sigma_{s}$ and $\Sigma_{u}$. Let $\Sigma_{s}$ denote the one which contains the origin (see Fig. 1). Then the followings hold:

(i) If all the eigenvalues of $K B$ are located in $\Sigma_{s}$, the closed-loop system (19) is practically stable.

(ii) If $K B$ has an eigenvalue in the region $\Sigma_{u}$, the closed-loop system (19) is not practically stable.

Some comments are put forth on Lemma 3. A necessary condition for the practical stability is that $I+K B$ is anti-Hurwitz. This is stronger than the necessary condition $\operatorname{det}[I+K B]>0$, which results from the Hurwitzness of $\bar{A}$ and the assumption $\operatorname{det}[-A]>0$. A sufficient condition is that $K B$ is Schur. In this connection, it is noted that when the derivative feedback is 


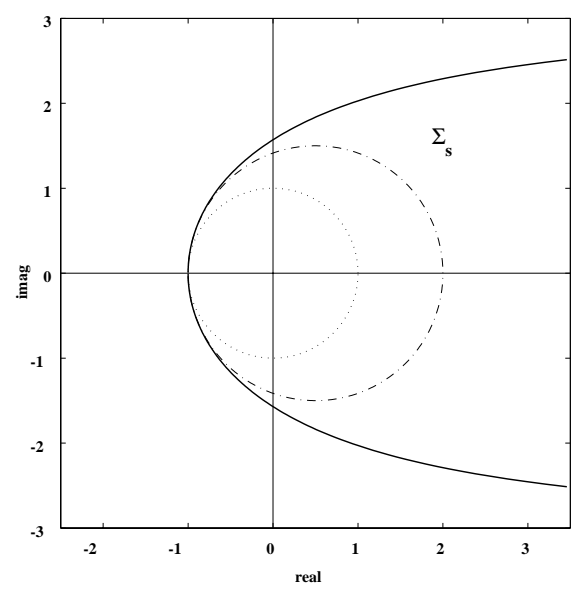

Fig. 1. Boundaries of $\Sigma_{s}$ (solid) and the unit disk(dotted)

accompanied with a small delay $\tau$,

$$
u(t)=-K \delta \dot{x}(t-\tau),
$$

the closed-loop system becomes of neutral type. The instability caused by such a small delay in the loop has been studied for a various kind of systems (see e.g., Barman et al. 1973; Datko 1988; Louisell 1995). Owing to the improved tool of Logemann et al. (1996), we know that the neutral system is practically stable if $K B$ is Schur. Conversely if $\left|\lambda_{i}(K B)\right|>1$ for some $i$, then the neutral system is not practically stable.

Next we examine the practical stability condition for the case of taking the sampled-data control approach. Supposing a sampling interval $T$ and the input generated by the zeroth-order hold, we have a sampled-data system,

$$
x_{i+1}=A_{s} x_{i}+B_{s} u_{i}, \quad x_{i}=\delta x(i T), u_{i}=u(i T),
$$

where $A_{s}=e^{A T}, B_{s}=\int_{0}^{T} e^{A t} B d t$. Take the approximate input $u_{i}=\frac{K}{T}\left(x_{i}-x_{i-1}\right)$, then the order of the closed-loop system becomes twice that of the plant. Simple analysis shows that as $T \rightarrow 0$, characteristic roots tend to $n$-tuple of 1 and $n$ eigenvalues of $-B K$. Further inspection shows that for $T>0$, the $n$-tuple roots are bifurcated as follows:

$\mu_{i}=1+\lambda_{i}\left((I+B K)^{-1} A\right) T+o(T), i=1, \ldots, n$.

Since $\bar{A}=(I+B K)^{-1} A$ is Hurwitz, $\mu_{i}$ belong to the open unit disk for small $T>0$. Thus the practical stability of the sampled-data system depends again on whether $B K$ (or equivalently $K B$ ) is Schur. It should be emphasized that the practical stability of the difference approximation is less restrictive than that of the sampled-data implementation, and that of the derivative feedback with a small delay.

Now we are in position to examine the case of using the dynamic controller (10) . When $D=0$, the closedloop system has been shown to be expressed by (13) .

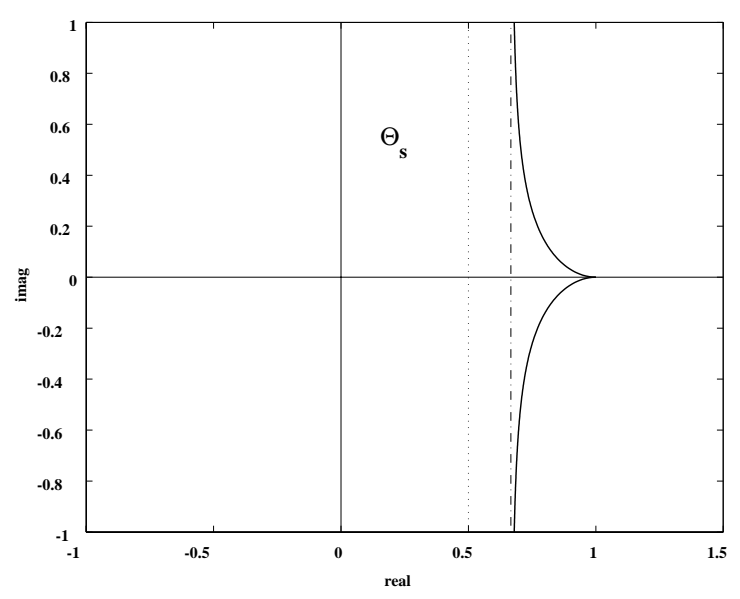

Fig. 2. Boundary of $\Theta_{s}$ (solid)

By applying Lemma 3, we know that the practical stability of the difference feedback controller simply depends on $\mathcal{K B}=-C B D_{c}$. Else if $D \neq 0$ and $D_{c}=0$, the practical stability depends on $\mathcal{K B}=-D C_{c} B_{c}$.

The graphical test based on the eigenvalues of $\Gamma(\infty)$ is more suited to the analysis of observer-based controllers. Figure 2 illustrates the region $\Theta_{s}$ which corresponds to $\Sigma_{s}$ in Fig. 1. The open region $\Theta_{s}$ is left to the boundary $\{\theta(j \omega):-2 \pi<\omega<2 \pi\}$, where $\theta(s)=s /\left(s-1+e^{-s}\right)$. It is of course that if $\lambda_{i}(\Gamma(\infty)) \in \Theta_{s}$, the practical stability is assured. The boundary asymptotically approaches the line that is parallel to the imaginary axis with passing through $\frac{2}{3}+j 0$. The asymptote is denoted by the dashed line in Fig.2, and the dashed line in Fig. 1 corresponds to the asymptote. Thus we have a simple sufficient condition for the practical stability.

\section{Lemma 4 If}

$$
\operatorname{Re} \lambda_{i}(\Gamma(\infty))<\frac{2}{3}, \quad i=1, \ldots, m,
$$

then the closed-loop system (19) is practically stable.

\section{OBSERVER-BASED CONTROLLERS}

To begin with, we analyze the full-order observer-based controllers. The following is a slight extension of Theorem 5 of Kokame et al. (2001).

Theorem 5 Suppose that $L$ and $K$ are such that $A-L C A$ and $A-B K$ are Hurwitz. Then the multiinput multi-output system (11) is stabilized practically by the full-order observer-based output difference controller,

$$
\begin{aligned}
\dot{\eta}(t) & =A \eta(t)+B u(t)+L\left(\frac{1}{T}(y(t)-y(t-T))\right. \\
& -C A \eta(t)-C B u(t)-D \dot{u}(t)) \\
u(t) & =-K \eta(t)
\end{aligned}
$$


if

$$
\lambda_{i}(D K L) \in \Theta_{s}, \quad i=1, \ldots, q .
$$

Especially if $D=0$, the practical stability is assured.

Proof: The closed-loop stability is a standard fact when applying the full-order observer-based controller:

$$
\begin{aligned}
\dot{\eta}(t) & =A \eta(t)+B u(t) \\
& +L(\delta \dot{y}(t)-C A \eta(t)-C B u(t)-D \dot{u}(t)) \\
u(t) & =-K \eta(t) .
\end{aligned}
$$

Notice that this controller is strictly proper:

$$
\begin{aligned}
& \dot{\eta}(t)=A_{c} \eta(t)+B_{c} \delta \dot{y}(t), \\
& A_{c}=(I-L D K)^{-1}(I-L C)(A-B K), \\
& B_{c}=(I-L D K)^{-1} L, \\
& u(t)=-K \eta(t) .
\end{aligned}
$$

As $D_{c}=0$, the closed-loop system (14) is stable. From (14), we have $\mathcal{K B}=-D C_{c} B_{c}=D K L(I-$ $D K L)^{-1}$. Manipulation yields that $\Gamma(\infty)=D K L$, hence Lemma 3 leads to the conclusion. Q.E.D.

If the triplet $(A, B, C)$ is controllable and observable, and further $\operatorname{det}[A] \neq 0$, then so is the triplet $(A, B, C A)$. Thus there always exist $L$ and $K$ that satisfy the assumptions of Theorem 5 .

The controller (27) nearly acts as the state feedback $u=-K \delta x$. If one wishes a controller acting asymptotically as $u=-K \delta \dot{x}$, he may use the following controller:

$$
\begin{aligned}
& \dot{\eta}(t)=(A-L C) \eta(t)+(B-L D) \dot{u}+L \delta \dot{y}(t) \\
& u(t)=-K \eta(t),
\end{aligned}
$$

where $A-L C$ is Hurwitz. If the derivative of measurements should be avoided, we may have its difference counterpart:

$$
\begin{aligned}
\dot{\eta}(t)= & (A-L C) \eta(t)+(B-L D) \dot{u} \\
& +\frac{1}{T} L(y(t)-y(t-T)) \\
u(t)=- & K \eta(t) .
\end{aligned}
$$

For this case, $\mathcal{K B}=D K(I+B K-L D K)^{-1} L=$ $D K(I-\bar{L} D K)^{-1} \bar{L}$, where $\bar{L}=(I+B K)^{-1} L$. Then the practical stability can be checked using $\Gamma(\infty)=$ $D K \bar{L}=D(I+K B)^{-1} K L$.

Now we proceed to the minimal-order observer-based controller for the case $D=0$ :

$$
\begin{aligned}
& \dot{\eta}(t)=M \eta(t)+N u(t)+H \delta \dot{y}(t), \\
& \delta \hat{x}(t)=Q \eta(t)+R u(t)+S \delta \dot{y}(t), \\
& u(t)=-K \delta \hat{x}(t),
\end{aligned}
$$

where $\eta \in R^{n-q}$, and the matrices are assumed to be compatible with the vectors. If the matrices $S, Q, U$ and $H$ satisfy

$$
\begin{aligned}
& H C A-U A=-M U, \\
& S C A+Q U=I, \\
& R=-S C B, N=U B-H C B
\end{aligned}
$$

with $M$ being Hurwitz, then $\eta$ and $\hat{x}$ converge to $U \delta x$ and $\delta x$, respectively. The dynamical system (31) (32) is then called the minimal-order observer. This observer combined with the output $u=-K \delta \hat{x}$ is rewritten in the standard form:

$$
\begin{aligned}
\dot{\eta}(t) & =\left(M-N K(I+R K)^{-1} Q\right) \eta(t) \\
& +\left(H-N K(I+R K)^{-1} S\right) \delta \dot{y}(t), \\
u(t) & =-K(I+R K)^{-1} Q \eta(t) \\
& -K(I+R K)^{-1} S \delta \dot{y}(t) .
\end{aligned}
$$

When using the controller (33) (or (37) ), the closedloop system is asymptotically stable if and only if $A-B K$ is Hurwitz. It is noticed here that the overall system can be written in the form of a derivative feedback, as in (13) :

$$
\begin{aligned}
\dot{z}(t) & =\mathcal{A} z(t)+\mathcal{B} v(t) \\
& =\left[\begin{array}{cc}
A & -B K(I+R K)^{-1} Q \\
0 & M-N K(I+R K)^{-1} Q
\end{array}\right] z(t) \\
& +\left[\begin{array}{c}
-B K(I+R K)^{-1} S \\
H-N K(I+R K)^{-1} S
\end{array}\right] v(t), \\
v(t) & =-\mathcal{K} \dot{z}(t)=[C 0] \dot{z}(t)=\delta \dot{y}(t),
\end{aligned}
$$

where $z(t)=\left[\begin{array}{ll}\delta x(t)^{T} & \eta(t)^{T}\end{array}\right]^{T}$.

For this case, we have $\mathcal{K B}=C B K(I+R K)^{-1} S$. By noting $I+\mathcal{K B}=(I-C B K S)^{-1}$, it is immediate that $\Gamma(\infty)=(I+\mathcal{K} \mathcal{B})^{-1} \mathcal{K} \mathcal{B}=C B K S$. Thus we have the following.

Theorem 6 Assume that the MIMO plant (11) has $D=0$, and $C$ is of full row rank. Further assume that the dynamic controller (33) satisfies the conditions (34) (35) (36), and both $M$ and $A-B K$ are Hurwitz. Then the difference approximate controller,

$$
\begin{aligned}
& \dot{\eta}(t)=M \eta(t)+N u(t)+\frac{1}{T} H(y(t)-y(t-T)), \\
& \delta \hat{x}(t)=Q \eta(t)+R u(t)+\frac{1}{T} S(y(t)-y(t-T)) \\
& u(t)=-K \delta \hat{x}(t),
\end{aligned}
$$

practically stabilizes the system (11), if

$$
\lambda_{i}(C B K S) \in \Theta_{s}, \quad i=1, \ldots, q .
$$

Example 7 Consider a second-order system having

$$
A=\left[\begin{array}{ll}
0 & 1 \\
1 & 1
\end{array}\right], B=\left[\begin{array}{l}
0 \\
1
\end{array}\right], C=\left[\begin{array}{ll}
0 & 1
\end{array}\right], D=0
$$

Taking $U=U_{\ell}=\left[\begin{array}{ll}\ell & \ell-1\end{array}\right]$ yields $M=-\ell, N=-\ell^{2}$, $H=\ell^{2}+\ell-1, S=\left[\begin{array}{ll}1-\ell & \ell\end{array}\right]^{T}, Q=\left[\begin{array}{ll}1 & -1\end{array}\right]^{T}$, and $R=\left[\begin{array}{ll}\ell-1 & -\ell\end{array}\right]^{T}$. By denoting $K=\left[\begin{array}{ll}k_{1} & k_{2}\end{array}\right]$, the controller (37) is stabilizing if and only if $\ell>0$, $k_{1}>1$ and $k_{2}>1$. For our example the output variable is scalar, hence Theorem 6 guarantees the practical stability if $C B K S=k_{1}(1-\ell)+k_{2} \ell<1$. We have examined the practical stability of the difference 

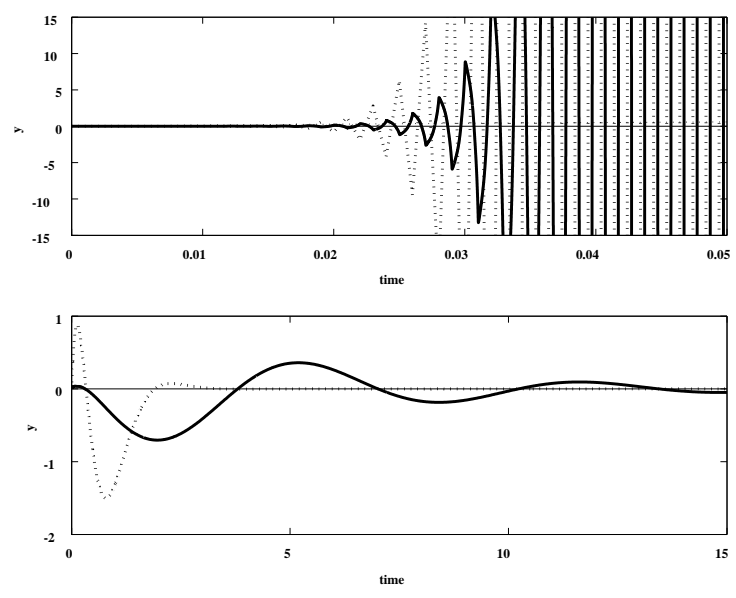

Fig. 3. Initial responses

approximate controller taking $\ell=2, k_{1}=2, k_{2}=1.3$, and $T=0.1$. The initial response is shown by solid line in the lower graph of Fig. 3. The dotted line is for reference, showing the response of the observer-based controller (37). The upper graph of Fig. 3 contains the response of the controller (37) having a small delay of $\tau=0.001$ (solid) in the measurement of $y$, and that of the sampled-data implementation with a sampling time $T=0.001$ (dotted). It is observed that the difference feedback is more robust than the sampleddata feedback. Further it has preserved the stability in the presence of the same delay in the measurements.

\section{CONCLUDING REMARKS}

The present paper has dealt with dynamic controllers which use the output differences in place of output derivatives. The practical stability of the closed-loop system is the main topic of the paper, and some stability criteria have been presented for the case of observerbased controllers. The criteria are written in a form of algebraic condition about the gain matrices. Taking the practical stability into account in the design process is left to the future work. This study was partly supported by Grant-in-Aid(No. 16560392) for Scientific Research.

\section{REFERENCES}

Van Assche, V., M. Dambrine, J. F. Lafay, and J. P. RICHARD (1999). Some problems arising in the implementation of distributed-delay control laws; Proc. IEEE Conf. on Decision Contr., Phoenix, 4668-4672.

Barman, J. F., F. M. CAllier, and C. A. DesOER(1973). $L^{2}$-stability and $L^{2}$-instability of linear time-invariant distributed feedback systems perturbed by a small delay in the loop, IEEE Trans. Automat. Contr., 18, 479-484.

DATKO, R. (1988). Not all feedback stabilized hyperbolic systems are robust with respect to small time delays in their feedbacks, SIAM J. Contr. Optimiz., 26, 697-713.

Engelborghs, K., M. Dambrine, and D. Roose (2001). Limitations of a class of stabilization methods for delay systems, IEEE Trans. Automat. Contr., 46, 336-339.

Just, W., T. Bernard, M. Ostheimer, E. ReiBOLD, and H. BENNER (1997). Mechanism of time-delayed feedback control, Phys. Rev. Lett., 78, 203-206.

KoKame, H., K. HiRATA, K. Konishi, and T. Mori (2001). Difference feedback can stabilize uncertain steady states, IEEE Trans. Automat. Contr., 46, 1908-1913.

KokAme, H. and T. Mori (2002). Stability preserving transition from derivative feedback to its difference counterparts, Proc. of the 15th IFAC World Congress, Barcelona, CD-ROM.

Konishi, K. and H. KoKAmE (1998). Observerbased delayed-feedback control for discrete-time chaotic systems, Phys. Lett. A, 248, 359-368.

Logemann, H., R. REBARber, and G. Weiss (1996). Conditions for robustness and nonrobustness of the stability of feedback systems with small delays in the feedback loops, SIAM J. Contr. Optimiz., 34, 572-600.

Louisell, J. (1995). Absolute stability in linear delay-differential systems: Ill-posedness and robustness, IEEE Trans. Automat. Contr., 40, 12881291.

NAKAJIMA, H. (1997). On analytical properties of delayed feedback control of chaos, Phys. Lett. A, 232, 207-210.

O'Connor, D. A. and T. J. TARn (1983). On stabilization by state feedback for neutral differential difference equations, IEEE Trans. Automat. Contr., 28, 615-618.

PALMOR, Z. (1980). Stability properties of Smith dead-time compensator controllers, Int. J. Contr., 32, 937-949.

PyRAGAS, K. (1992). Continuous control of chaos by self-controlling feedback, Phys. Lett. A, 170, 421-428.

SAntos, O. and S. Mondie (2000). Control laws involving distributed time delays: Robustness of the implementation, Proc. Amer. Contr. Conf., Chicago, 2479-2480.

Ushio, T. (1996). Limitation of delayed feedback control in nonlinear discrete-time systems, IEEE Trans. Circuits Syst. I, 43, 815-816.

YAmамото, S., T. Hino, and T. Ushio (2000). A dynamic delayed feedback controller for chaotic discrete-time systems, Proc. Amer. Contr. Conf., Chicago, 3358-3359.

YANUSHEVSKY, R. T. (1992). On robust stabilization of linear differential-difference systems with unstable D-operator, IEEE Trans. Automat. Contr., 37, 652-653. 